\title{
FINITE ELEMENT ANALYSIS ON THE KEY COMPONENTS OF THE HORIZONTAL SEALING DEVICE FOR THE BULK MATERIAL PACKAGING MACHINE
}

\author{
Ning Tingzhou ${ }^{1}$, Zhang Yao ${ }^{2}$, Fu Ling ${ }^{1^{*}}$ \\ ${ }^{1}$ College of Mechanical and Electrical Engineering, Zaozhuang University, Zaozhuang 277160, China \\ ${ }^{2}$ College of Electrical Engineering, Changzhou Vocational Institute of Mechatronic Technology, Changzhou \\ 213164,China \\ E-mail:fuling841225@163.com
}

\begin{abstract}
Horizontal sealing plate is a key part of the horizontal sealing device of the bulk material packaging machine, and its force and heat have a great influence on the packaging effect. In order to study the force and heat of the horizontal sealing plate when it was working, the force and heat analysis was carried out based on SOLIDWORKS Simulation. The results showed that, (1) The stress on the horizontal sealing plate was relatively small, the maximum value was $348.6 \mathrm{MPa}$, which was less than the yield stress of $620.4 \mathrm{MPa}$ of alloy steel. The maximum displacement of the horizontal sealing plate was $6.426 \times 10^{-3} \mathrm{~mm}$, which was very small compared to the overall size of the horizontal sealing plate, and the influence on the working process could be ignored.(2) Each point of the horizontal sealing plate was evenly heated, but because part of the heat was lost during the transfer process, the average surface temperature was $134^{\circ} \mathrm{C}$, which was $1^{\circ} \mathrm{C}$ away from the actual optimal processing temperature. Therefore, in actual processing, the heating temperature was $1^{\circ} \mathrm{C}$ higher than the ideal packaging temperature. The above research results provide the theoretical basis and reference for the design of key parts of the bulk material packaging machine.
\end{abstract}

Keywords: Bulk material, Packaging machine, Horizontal sealing plate, Finite element analysis.

\section{Introduction}

Bulk materials such as sugar, salt and tea have become necessities in people's lives. The traditional packaging of bulk materials has been transformed from manual packaging to mechanized operations, which effectively improves the packaging efficiency of bulk materials[1-3]. The packaging machine plays an important role in the packaging of materials and has been widely used in various fields such as industry and agriculture[4-5].

The packaging process of each type of packaging machine is different, but all need to realize the packaging of the packaging material through the vertical sealing device and the horizontal sealing device. The horizontal sealing is usually carried out after the vertical sealing is completed[6]. The quality of the horizontal sealing will directly affect the storage of materials, the beauty of the packaging and the sales of the products. As a key component of the horizontal sealing device, the horizontal sealing plate has a great influence on the quality of the horizontal sealing[7].

In this paper, the key component of horizontal sealing device of bulk material packaging machine(the horizontal sealing plate) is taken as the research object.
The finite element analysis method[8-12]is used to analyze its stress and heat based on SOLIDWORKS simulation, so as to check whether it can meet the working requirements, and provide theoretical basis and reference for the design of horizontal sealing plate.

\section{Structure and Working Principle of the Horizontal Sealing Device}

The structure of the horizontal sealing device of the bulk material packaging machine is shown in Figure 1. The power of the horizontal sealing device is transmitted by the cam 1 on the transmission shaft, and the rolling bearing 6 connected with the cam 1 is driven by the thrust of the cam 1 , so as to promote the movement of the horizontal sealing plate 4 and realize the packaging of materials. At the same time, a cam with the same structure and motion on the upper side of the cam which pushes the heating plate to move is also provided, and the damping spring 2 is pressed backward through the bearing to ensure the stability of the whole movement. When the two cams return, the damping springs on both sides spring up, and the heating plates separate to complete the whole packaging process. 


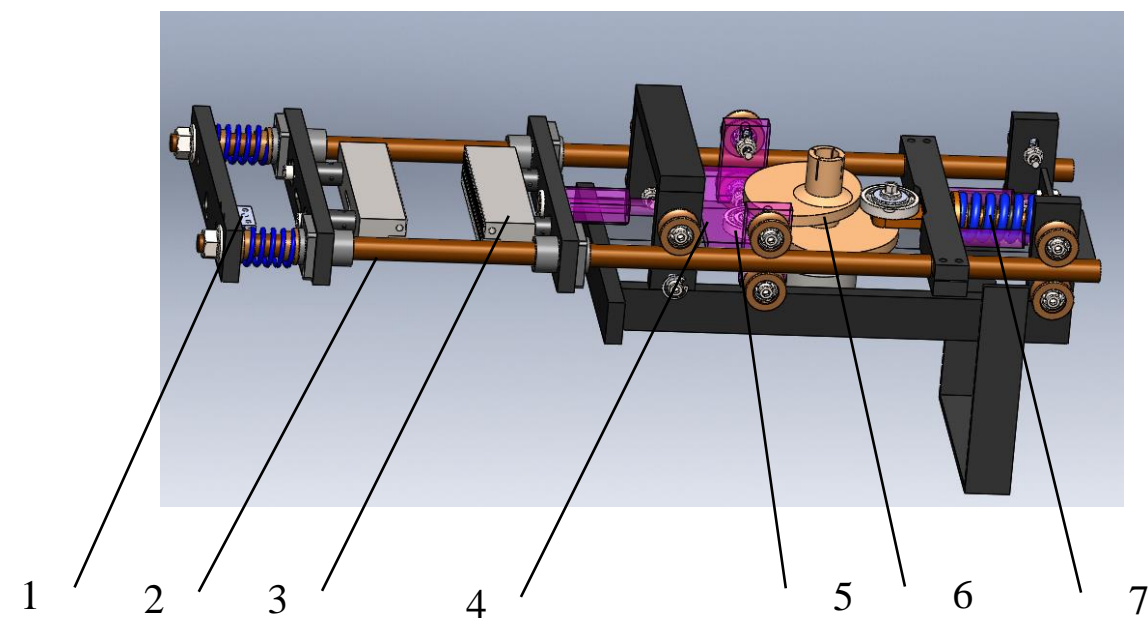

1.Thrust plate, 2. Sliding guide rod, 3. Horizontal sealing plate, 4. Fixed plate of rolling bearing, 5.Rolling bearing, 6. Cam, 7. Damping spring

Figure 1: Horizontal sealing device of bulk material packaging machine

It can be seen from Figure 1 that the whole device is transmitted by the transmission shaft, which fundamentally ensures the consistency of the movement of blanking, feeding and packaging, and ensures the continuous working capacity of the bulk material packaging machine. The pushing part of the horizontal sealing device mainly adopts the cam and rolling bearing to cooperate. The sealing method is simple in structure. The cam is adopted to ensure that the axial movement is changed to the axial plane motion. The symmetrical shape of the cam ensures the smooth movement of the entire packaging process. The bearing matched with the cam is a standard part and successfully avoids the shortcomings of easy wear of the cam, and the bearing as a standard part is easy to replace. Springs are added at both ends of the horizontal sealing device, which can buffer and absorb shock, especially the right spring part also has a cam with the same structure as the driving sealing device, which greatly improves the shock absorption function of the horizontal seal device and ensures the stability of the transmission shaft movement. Since the transmission shaft drives the movement of the entire packaging machine, this design also improves the working stability and reliability of the entire bulk material packaging machine.

\section{Factors Affecting Heat Sealing of Horizontal Sealing Device}

There are many factors that affect the sealing quality of the horizontal sealing device of the packaging machine. The main factors are the different sealing materials used by the packaging machine, and whether the performance of the packaging materials is stable under the condition of heating, etc. When a certain packaging material is determined during the production of packaging, the main factors that affect the packaging are as follows: the temperature of the heating plate during heat sealing, the pressure on both sides of the packaging material during sealing, and the heating method adopted by the heating plate, etc[13].

\subsection{Heat Sealing Temperature}

In the process of product packaging and sealing, the product packaging materials need to be heated at a certain temperature. The main purpose is to gradually change the packaging materials into viscous flow state to ensure that the sealing has enough strength. Heating at a certain temperature refers to the heat sealing temperature. Most of the packaging materials are polymers without accurate melting point, because they have only one temperature change amplitude. When the heating temperature reaches between the temperature change amplitude, the packaging material will change from solid state to viscous flow state. Therefore, in the process of packaging, the heat sealing temperature should be less than the decomposition temperature of packaging materials, and also greater than the brittleness rising temperature of polymers. The above two temperature ranges also affect the difficulty of heat sealing.

In the process of heat sealing, when the temperature of heat sealing is too high, the packaging materials may be damaged, burned out or the heating plate may loosen on the horizontal sealing device, which will affect the whole packaging process. When the temperature is too high, it will damage the material at the packaging place and affect the packaging. When the heat sealing temperature is too low, the packaging material is heated unevenly, and some sealing materials may not become viscous flow state, which is difficult to 
ensure the sealing quality[14]. Therefore, in the process of product packaging and sealing, the heat sealing temperature must be selected reasonably, neither too high nor too low.

\subsection{Heat Sealing Pressure}

The function of heat sealing pressure is to heat the polymer materials at the sealing place to the molten state, and make the packaging materials diffuse each other in the molten state through appropriate pressure, so as to make each polymer close to each other and produce force. When the heat sealing pressure is too high, the force between the polymers will be reduced, which will affect the packaging quality. When the packaging temperature is too low, the sealing may not be firm. Therefore, when packaging products, we must choose a reasonable heat sealing pressure[15].

\subsection{Heat Sealing Time}

The heat sealing time mainly refers to the residence time of the heating plate when it heats the sealing material. In other words, the heat sealing time plays a crucial role in the quality of heat sealing. A longer heat sealing time can make the material at the sealing place more evenly heated, and also make the polymer material in the viscous flow state have better viscosity and enhance the sealing strength[16].

\section{Finite Element Analysis of Key Components of Horizontal Sealing Device}

The horizontal sealing device plays a vital role in the bulk material packaging machine, and the performance of the horizontal sealing plate as a key component of the horizontal sealing device also plays an important role in the entire packaging machine. In the entire product packaging process, the horizontal sealing plate is the main force and heating element in the entire packaging mechanical device. Therefore, its various performances have an important impact on the quality and efficiency of the packaging machine. Therefore, it is necessary to verify the static calculation example of the horizontal sealing plate of the horizontal sealing device[17].

\subsection{Stress Analysis}

(1) Load the horizontal sealing plate and Start SOLIDWORKS Simulation in SOLIDWORKS Add-Ins. First, click File, Open, and then select the horizontal sealing plate (as shown in Figure 2). Second, click Tools, Add-Ins, and then check SOLIDWORKS Simulation.

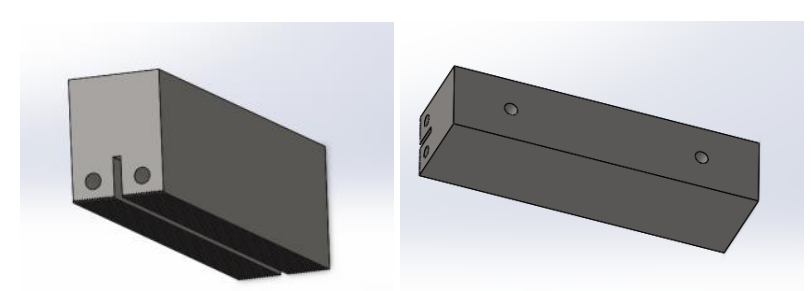

Figure 2: Three-dimensional model of horizontal sealing plate

(2) Study selection. Click the Simulation tab, select the Static in New Study, and then confirm.

(3) Material application. The part needs to be given the corresponding material. Click the Simulation tab, Apply Material, select Alloy Steel, click Apply, and then click Close.

(4) Fixtures advisor. According to the actual assembly situation, the appropriate fixture is selected in the simulation experiment. Click the Simulation tab, Loads/ Fixtures, Fixtures, Fixed Geometry. Because the horizontal sealing plate is connected by screws, the two threaded hole surfaces on the horizontal sealing plate should be selected when selecting the fixed stress surface of the horizontal sealing plate.

(5) External Loads Advisor. According to the actual working condition of the parts, the loads to be applied in the simulation is determined. First, click the Pressure in External Loads Advisor. According to the actual packaging, the maximum pressure of the horizontal sealing plate is $3 \times 10^{5} \mathrm{~N} / \mathrm{m}^{2}$, and when the two horizontal sealing plates contact, the main stress surface is each inclined plane, and the direction of the contact force is the normal direction of each inclined plane. Second, click the temperature in External Loads Advisor. Considering that the heating plate needs to be heated during the actual contact process, the temperature load is loaded in the through hole where the heating device is placed, and the temperature is $135^{\circ} \mathrm{C}$. The loading methods are shown in Figure 3.

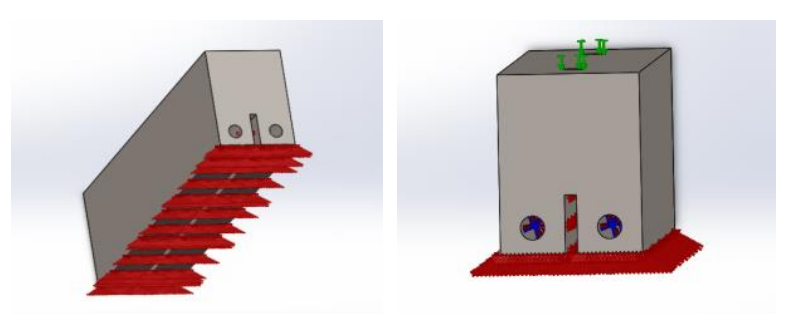

$\begin{array}{ll}\text { (a) Pressure load } & \text { (b) Temperature load }\end{array}$ Figure 3: Load application position

(6) Mesh creation. As we know, the finite element analysis mainly uses the method of dividing the element, that is, the whole research object is divided into a finite number of small elements by using the method of simulation analysis. 
Through the study of small elements, the overall deformation and other related research data are obtained. The specific operations are as follows: click Mesh, the finest Mesh Density, and click OK. The result is shown in Figure 4.

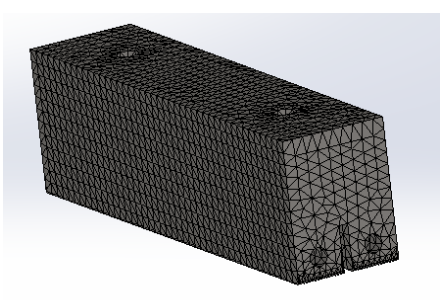

Figure 4: Mesh division of the horizontal sealing plate under stress analysis

(7) Run This Study. This step is to summarize and analyze the simulated conditions, and finally output the results, as shown in Figure 5, Figure 6, and Figure 7.

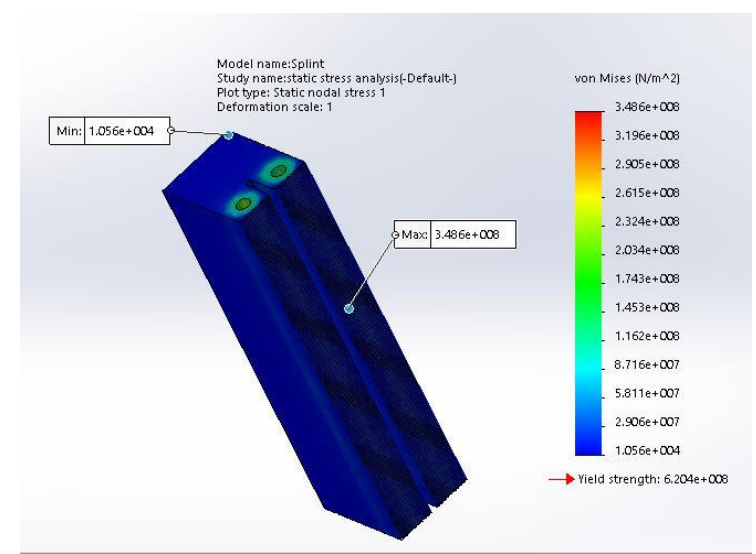

Figure 5: Stress distribution diagram of horizontal sealing plate

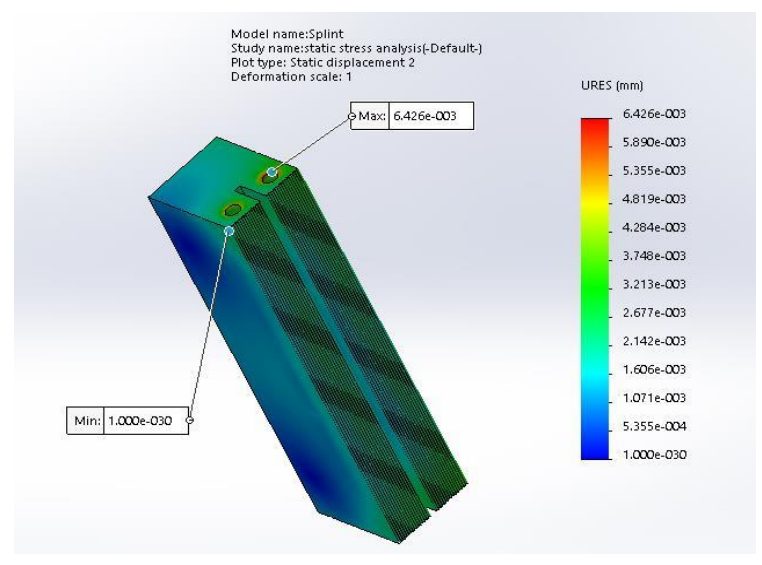

Figure 6: Displacement distribution diagram of horizontal sealing plate

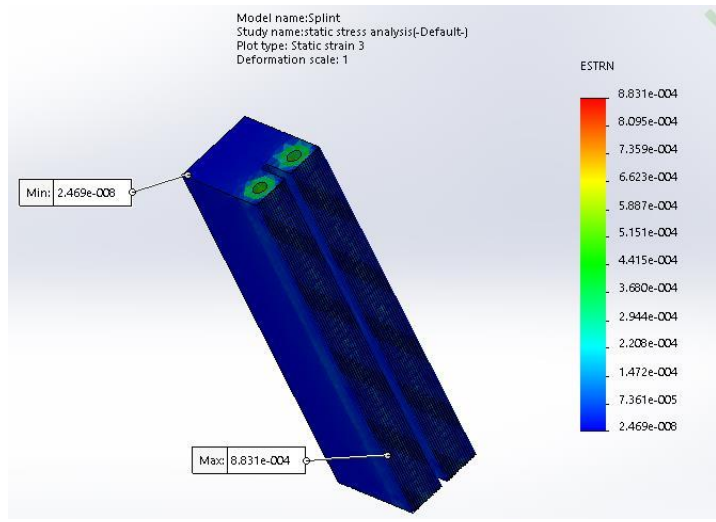

Figure 7: Strain distribution diagram of horizontal sealing plate

The above static analysis is mainly to check the stiffness of the horizontal sealing plate, which refers to the ability of an object to resist elastic deformation when deformed by an external force. Through the finite element analysis of the horizontal sealing plate, the force and deformation of the horizontal sealing plate under working loads can be obtained. It can be seen from Figure 5 that the stress when the material yields is $620.4 \mathrm{MPa}$, and the simulation shows that the maximum stress generated when the horizontal sealing plate receives the maximum force during its work is $348.6 \mathrm{Mpa}$, which is far lower than the yield limit of the material. Figure 6 shows that the maximum displacement of the horizontal sealing plate is $6.426 \times 10^{-3} \mathrm{~mm}$, and its maximum deformation is very small for the horizontal sealing plate. Therefore, the displacement and strain produced by the horizontal sealing plate are within the elastic range, that is, the material can recover its original shape. Therefore, the design of the horizontal sealing plate can meet the needs of the normal operation of the packaging machine.

\subsection{Thermal Analysis}

In the process of packaging products, the horizontal sealing plate needs to heat the material of the packaging bag. Therefore, when performing finite element analysis on the horizontal sealing plate, the influence of temperature on the horizontal sealing plate needs to be considered, so it needs to carry out the thermal analysis. The specific steps are as follows:

(1) Model loading and study selection. Open the 3D model of the horizontal sealing plate separately, click the Simulation tab, select the Thermal in New Study.

(2) Material application. The step is the same as the Static, and the Alloy Steel material is selected. 
(3) External Loads Advisor. Since the packaging bag needs to be heated and plasticized in the actual processing process, and generally the best heating temperature is $135{ }^{\circ} \mathrm{C}$, and because the heating rod holes of the horizontal sealing plate are directly in contact with the heating rods, the temperature of $135{ }^{\circ} \mathrm{C}$ should be selected first when applying heat to them. For the processing of small-volume products such as bulk materials, they are often carried out in the constant temperature workshop, so $22{ }^{\circ} \mathrm{C}$ is selected as the convection option. The thermal effect is shown in Figure 8.

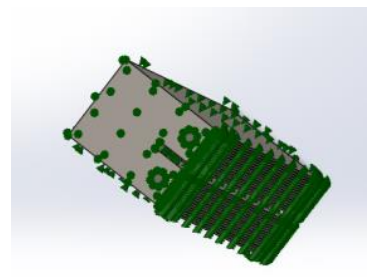

(a) Convective heat load

(b) Temperature heat load

Figure 8: Thermal effect diagram

(4) Meshing Creation. The specific step is the same as the Static, try to choose the finest mesh density to achieve more accurate output results, as shown in Figure 9.

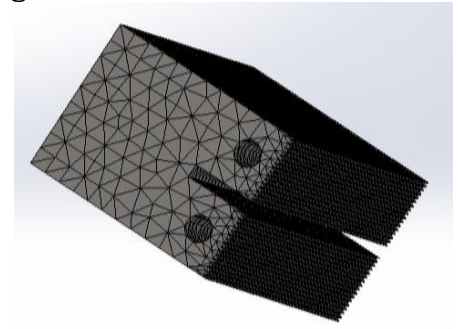

Figure 9: Mesh division of the horizontal sealing plate under thermal analysis

(5) After completing the above settings, run this study, and the result is shown in Figure 10.

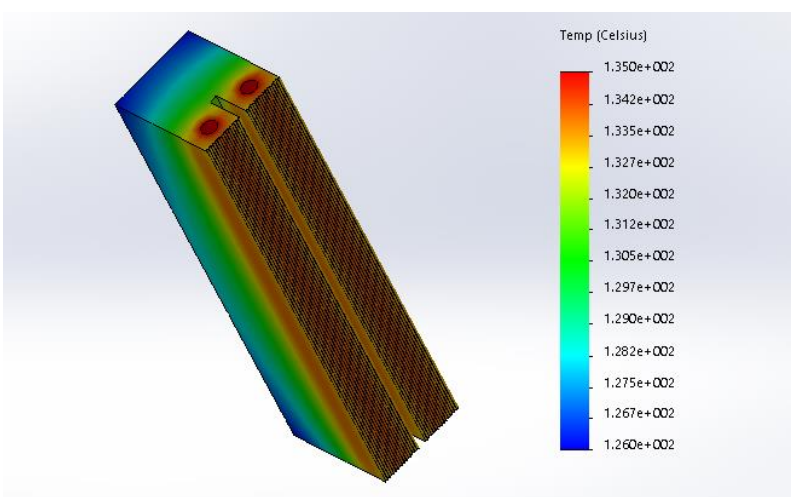

Figure 10: Thermal analysis result
As shown in Figure 11, after the simulation, the temperature at each horizontal sealing line of the horizontal sealing plate was detected, and it was found that the temperature at each point was uniform. Because part of the heat was lost during the transfer process, so the average surface temperature is $134{ }^{\circ} \mathrm{C}$, which is $1{ }^{\circ} \mathrm{C}$ different from the actual optimum processing temperature. In order to make the horizontal sealing plate reach the best packaging temperature, the simulated temperature was adjusted to $136^{\circ} \mathrm{C}$. The result was shown in Figure 12. The average temperature at each point of the horizontal sealing plate is $135.05^{\circ} \mathrm{C}$, which had reached the best temperature for normal packaging of the packaging material. Therefore, in actual processing, the heating temperature is $1^{\circ} \mathrm{C}$ higher than the ideal packaging temperature.

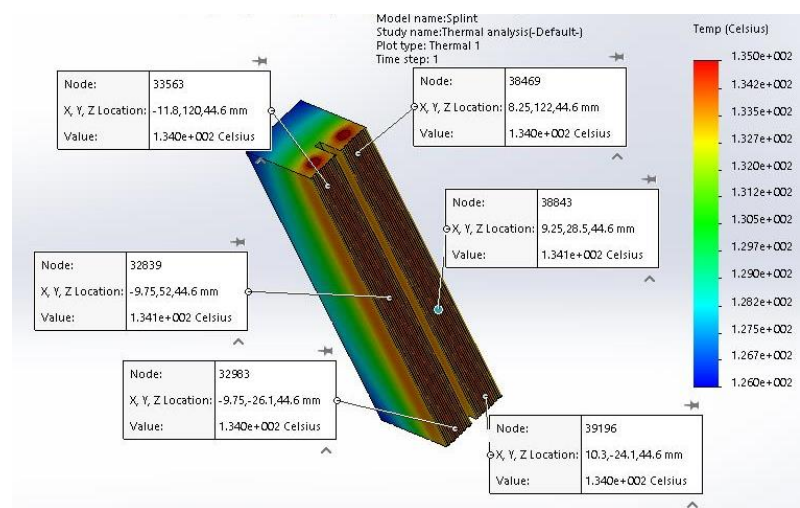

Figure 11: Detection diagram of each point of horizontal sealing plate under thermal analysis- First regulation simulation

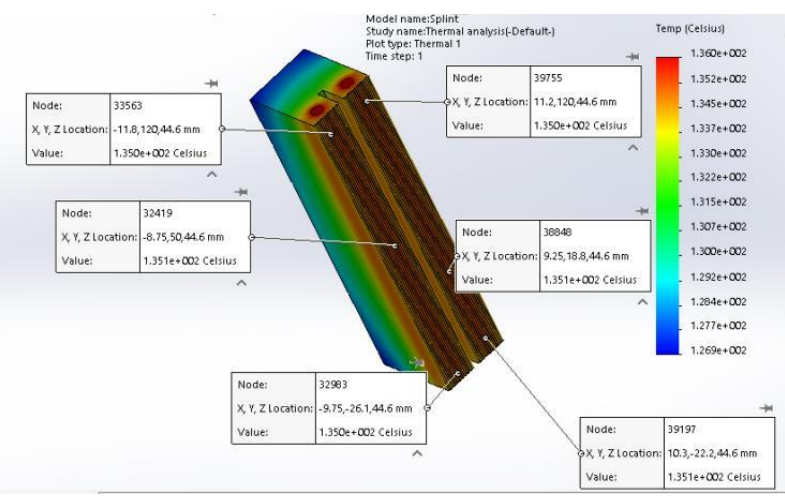

Figure 12: Detection diagram of each point of horizontal sealing plate under thermal analysisSecond regulation simulation

\section{Conclusions}

Based on SOLIDWORKS Simulation, the stress and thermal analysis of the key components of the horizontal sealing device for the bulk material packaging machine were carried out. The results showed that the designed horizontal sealing plate could meet the actual needs. 


\section{References}

[1] FENG Hui-ping, HU Ya-nan, YAN Chen-yu, et al. (2017). Design of control system for multifunctional tea packaging machine based on TIA portal. Food \& Machinery,33(7), pp.85-88.

[2] YUAN Fei. (2020). Based on Solidworks Chocolate Packaging Machine Structure Design. The Food Industry,41(11),pp.232-236.

[3] ZUO Bin, ZHANG Yongkang, GUO Nanchu.(2020). Design and Simulation Study of Multifunctional Apple Picking and Packing Machine. Research and Exploration in Laboratory,39(9),pp.108-113.

[4] ZHOU Dan, XIA Rufei, LI Gan, et al.(2020). Design of ultra-fine powder cleaning valve bag packaging machine. China Powder Science and Technology,26(6), pp.25-30.

[5] YUAN Fei, GAO Xue. (2020). Design and Development of Automatic Food Packaging Machine. The Food Industry, 41(5),pp.220-223.

[6] Li Wenfang, Fu Ruiling, Yu Chunlai. (2020). Speed Control of Transverse Sealing and Cross Cutting Mechanism of Food Packaging Machine. Packaging and Food Machinery,38(4),pp.66-69.

[7] Chenyu Yan.(2017). Study on The Design of Multifunctional Tea Packaging Machine. Shaanxi University of Science \& Technology.

[8] LIN Huan, XU Linyun, XUAN Yan, et al. (2020). Modal analysis and experimental study of the multistage Y-type ginkgo tree using the finite element method. Journal of Forestry Engineering,5(1),pp.148-155.

[9] XI Xi, YANG Yang, ZHANG Zhongfeng. (2020). Pullout force and finite element analysis of T-type components of Vitex negundo L. scrimber with different node forms.Journal of Forestry Engineering, 5(1),pp.182-187.

[10] TAN Xinjian, GUO Kejun, ZHANG Diansong, et al. (2019). Design of the lifting ramie decorticator. Journal of Forestry Engineering,4(3),pp.106-111.

[11] MA Yan, SONG Mingliang, YANG Chunmei, et al. (2019). Design and analysis of double-end planing and milling machine with automatic tool-exchange function for processing doors and windows. Journal of Forestry Engineering, 4(1),pp.108-114.

[12] LI Wei, GAO Ying, MENG Xinmiao, et al. (2020). Study on compressive performance of angel steel-glued laminated timber L-shaped composite column. Journal of Forestry Engineering,5(1),pp.53-60.

[13] Sun Yuejin. (2015). Analysis on the Solution to the Problem of Uneven Heat Sealing Strength of Packaging. Mechanical and Electrical Information, 26,pp.25-28.

[14] Zhang Hongjing, Zhang Guoqing, Wang Cuiqing. (2015). Failure Mode Analysis of Heat Sealing Strength of Plastic Packaging Bag. Packaging Journal,7(3),pp.27-31.

[15] NI Zheng-shun, ZHANG Yi-jing. (2002). Finite Element Analysis and Optimal Design in the Design of Packaging Machinery. Journal of Zhuzhou Institute of Technology,4, pp.5-6.

[16] Sun Minda, Zhang Xidong, Gao Xue. (2014). Research and Implementation of Heat Sealing Testing Experiment of Food Packaging Bags. Forest Engineering,30(1),pp.100-103.

[17] Qi Jian. (2019). SOLIDWORKS: Starting from basic education and taking root in China's manufacturing industry. Intelligent Manufacturing, 12,pp.11-12. 